\title{
First-line sunitinib or pazopanib in metastatic renal cell carcinoma: The Canadian experience
}

\author{
Aly-Khan A. Lalani, MD'; Haocheng Li, PhD2; Daniel Y.C. Heng, MD, MPH3; Lori Wood, MD'; Austin Kalirai5; \\ Georg A. Bjarnason, MD'; Hao-Wen Sim, MBBS, BMedSci'; Christian K. Kollmannsberger, MD;; \\ Anil Kapoor, MD'; Sebastien J. Hotte, MD'; Marie Vanhuyse, MD ${ }^{10}$; Piotr Czaykowski, MD, MSc ${ }^{11}$; \\ M. Neil Reaume, MD'12; Denis Soulieres, MD, MSc'33; Peter Venner, MD'; Scott North, MD, MHPE'; \\ Naveen S. Basappa, MD'
}

'Cross Cancer Institute, University of Alberta, Edmonton, AB; ${ }^{2}$ Departments of Oncology \& Community Health Sciences, University of Calgary, Calgary, AB; ${ }^{3}$ Tom Baker Cancer Centre, University of Calgary, Calgary, AB; ${ }^{4}$ Queen Elizabeth II Health Sciences Centre, Halifax, NS; ${ }^{5}$ Faculty of Sciences, University of Alberta, Edmonton, AB; ${ }^{\circ}$ Sunnybrook Odette Cancer Centre, University of Toronto, Toronto, ON; ${ }^{7}$ Princess Margaret Cancer Centre, University Health Network, University of Toronto, Toronto, ON; ${ }^{8 B C}$ Cancer Agency Vancouver Cancer Centre, Vancouver BC; ${ }^{9}$ Juravinski Cancer Centre, Hamilton, ON; ${ }^{10}$ Montreal General Hospital, McGill University, Montreal, QC; ${ }^{11}$ Cancer Care Manitoba, Winnipeg, MB; ${ }^{12 T h e ~ O t t a w a ~ H o s p i t a l ~ C a n c e r ~ C e n t r e, ~ O t t a w a, ~ O N ; ~}{ }^{13}$ Centre Hospitalier de I'Université de Montréal, Montreal, QC; Canada

Cite as: Can Urol Assoc J 2017; 11 (3-4):112-7. http://dx.doi.org/10.5489/cuaj.4398

\section{Abstract}

Introduction: Clinical trial data has shown pazopanib to be noninferior in overall survival (OS) compared to sunitinib as first-line treatment for metastatic renal cell carcinoma (mRCC). The purpose of this study was to evaluate outcomes and compare dose-modifying toxicities of mRCC patients treated with suntinib or pazopanib in the real-world setting.

Methods: Data were collected on mRCC patients using the prospective Canadian Kidney Cancer Information System (CKCis) database from January 2011 to November 2015. Statistical analyses were performed using Cox regression adjusted for several risk factors and the Kaplan-Meier method.

Results: We identified 670 patients treated with sunitinib $(n=577)$ and pazopanib $(n=93)$. There were no significant differences in International Metastatic Renal Cell Carcinoma Database Consortium (IMDC) risk groups $(p=0.807)$. Patients treated with sunitinib had improved OS compared with pazopanib (median 31.7 vs. 20.6 months, $p=0.028$; adjusted hazard ratio [aHR] 0.60; 95\% confidence interval $[\mathrm{Cl}]$ 0.38-0.94). Time to treatment failure (TTF) was numerically, but not statistically, improved with sunitinib (medians $11.0 \mathrm{vs}$. 8.4 months, $\mathrm{p}=0.130$; aHR $0.87 ; 95 \% \mathrm{Cl} 0.59-1.28)$. Outcomes with individualized dosing on sunitinib were unavailable for this analysis. Patients treated with sunitinib had a higher incidence of mucositis, hand-foot syndrome, and gastroesophageal reflux disease; patients treated with pazopanib had a higher incidence of hepatotoxicity.

Conclusions: In Canadian patients with mRCC, treatment with sunitinib appears to be associated with an improved OS compared to pazopanib in the first-line setting. Patient selection factors and the contemporary practice of individualized dosing with sunitinib may contribute to these real-world outcomes and warrant further investigation.

\section{Introduction}

Vascular endothelial growth factor (VEGF) is a key pathway involved in tumour angiogenesis and plays a significant role in the progression of renal cell carcinoma (RCC). The treatment landscape of metastatic RCC (mRCC) has rapidly evolved over the last decade, transitioning out of the cytokine era and into the era of VEGF tyrosine-kinase inhibitor targeted therapy (VEGF-TT). Standard first-line VEGF-TT begins with sunitinib or pazopanib. Sunitinib has been shown to have a significantly longer progression-free survival (PFS, 11 vs. 5 months) and a numerically better overall survival (OS, 26.4 vs. 21.8 months; $p=0.051$ ) compared with interferon. ${ }^{1,2}$ Pazopanib has shown a significant increase in median PFS (9 vs. 4 months), but not a significantly improved OS (23 vs. 21 months; hazard ratio [HR] $0.91 ; 95 \%$ confidence interval [CI] 0.71-1.16) when compared with placebo. ${ }^{3}$ A head-tohead prospective trial, COMPARZ, has shown pazopanib to be non-inferior to sunitinib in PFS (8.4 vs. 9.5 months. HR 1.05. 95\% Cl 0.90-1.22) and OS (28.4 vs. 29.3 months; HR $0.92 ; 95 \% \mathrm{Cl} 0.79-1.06)$ with better health-related quality of life scores. ${ }^{4,5}$ Another trial has shown that toxicity can influence patient and clinician preference for pazopanib over sunitinib. ${ }^{6}$ However, these trials used sunitinib on the classic schedule of four weeks on treatment, followed by two weeks off treatment (in a six-week cycle) and did not allow for the treatment schedule alterations, which are now increasingly used outside clinical trials.

Studies have shown a pharmacokinetic and clinical rationale for using non-classic, or alternative, dosing schedules with sunitinib. ${ }^{7-11}$ This spurred our interest in investigating the outcomes of patients with mRCC treated with VEGFTT in Canada, where dose individualization with alternate 
First-line therapy in metastatic RCC: Canadian experience

schedules of sunitinib is commonly used. A recent retrospective analysis by the International Metastatic RCC Database Consortium (IMDC) of patients treated with first-line sunitinib or pazopanib showed similar survival outcomes. ${ }^{12}$ However, this study did not capture the practice of alternate dosing schedules on sunitinib and did not report on patient toxicities. Real-world data that reflects the contemporary experience with VEGF-TT, and the concomitant adverse events seen in an unselected patient setting, has been lacking.

In this analysis, we use a prospective, population-based database to investigate survival outcomes and dose-modifying toxicities of patients with mRCC in Canada, where dose individualization of sunitinib is more prevalent.

\section{Methods}

\section{Study design}

We retrieved data from the prospective Canadian Kidney Cancer Information System (CKCis) database, which consists of patients from 14 academic centres across Canada, from January 2011 to November 2015. The participating centres represent sites where the vast majority of patients with $\mathrm{mRCC}$ are treated in Canada and capture the national practice pattern: British Columbia Cancer Agency; Cross Cancer Institute and Tom Baker Cancer Centre (Alberta); Cancer Centre Manitoba; Princess Margaret Cancer Centre, Sunnybrook Odette Cancer Centre, Juravinski Cancer Centre, The Ottawa Hospital Cancer Centre, London Regional Cancer Centre, and St. Joseph's Health Centre (Ontario); Centre Hospitalier de I'Université de Montréal, Montreal General Hospital, and Centre Hospitalier Universitaire de Quebec (Quebec); Queen Elizabeth II Health Sciences Centre (Nova Scotia). Patients had provided consent for entry into $\mathrm{CKCis} \mathrm{prospec-}$ tively and all centres have research ethics board approval for $\mathrm{CKCis} \mathrm{projects.} \mathrm{All} \mathrm{research} \mathrm{was} \mathrm{conducted} \mathrm{according} \mathrm{to}$ the principles of the Declaration of Helsinki. Patients diagnosed with mRCC treated with first-line sunitinib or pazopanib were included. Patients undergoing primary surgery or metastatectomy were eligible. Patients were excluded if they received interferon or another systemic therapy in the first-line setting.

Baseline demographic, clinical, and laboratory data were collected. The decision to start sunitinib or pazopanib and choice of specific agent was at the discretion of the treating physician. Treatment-associated toxicities were defined and evaluated according to the Common Terminology Criteria for Adverse Events, version 3.0, where CKCis captures reasons for dose-modification based on toxicities of Grade 3 or 4, or multiple Grades 1 and 2.

\section{Treatment outcomes}

OS was defined as the time from initiation of first-line therapy to death from any cause. Time to treatment failure (TTF) was defined as time from initiation of first-line therapy to date of discontinuation or death from any cause, whichever came first.

\section{Statistical analysis}

The primary objective was to assess OS in patients with mRCC treated with sunitinib or pazopanib. TTF and dosemodifying toxicities were secondary endpoints. Distributions of OS and TTF were calculated using the Kaplan-Meier method. Median OS and TTF, along with 95\% Cls were reported. Associations between OS and TTF were assessed using the Wald chi-square test from Cox regression in multivariable analysis, adjusted for sex and IMDC risk factors: low performance status (Karnofsky performance score [KPS] $<80 \%$ ), low hemoglobin, elevated corrected calcium, elevated neutrophils, elevated platelets, and time from diagnosis to treatment $<1$ year. ${ }^{13}$ Age was treated as a continuous variable. Subgroup efficacy analyses were performed by IMDC risk groups. Fisher's exact tests were used to compare toxicities between the two therapies.

\section{Results}

\section{Patient and disease characteristics}

Overall, our cohort consisted of 670 patients as shown in Table 1 . The median age was 65 years and the majority of patients were male with good performance status. Most patients underwent prior nephrectomy (81.8\%). Eighty-four percent of patients had clear cell histology and there was no significant difference between both groups regarding patients with non-clear cell histology $(16.3 \%$ in sunitinib group vs. $12 \%$ in pazopanib group; $p=0.356$ ). With regard to IMDC risk groups, the majority of patients had intermediaterisk disease $(57.9 \%)$ and the groups were well-balanced overall between the two therapies.

\section{Treatment exposure}

Patients received treatment in the first-line setting with either sunitinib $(n=577)$ or pazopanib $(n=93)$. The proportion of patients starting on full dose of both treatments was similar (85\% for sunitinib and $83 \%$ for pazopanib). Regarding subsequent treatment, $42.9 \%$ of patients overall received second-line therapy (axitinib, everolimus, or other) and this was similar between the sunitinib and pazopanib groups $(43.5 \%$ vs. $40 \%$, respectively; $p=0.709)$. 
Lalani et al.

\begin{tabular}{lccc}
\hline Table 1. Baseline patient and disease characteristics & \\
\hline Characteristic, $\mathbf{n}(\%)$ & $\begin{array}{c}\text { Pazopanib } \\
\text { (n=93) }\end{array}$ & $\begin{array}{c}\text { Sunitinib } \\
\text { (n=577) }\end{array}$ & p \\
\hline $\begin{array}{l}\text { Median age, years (IOR) } \\
\text { Gender }\end{array}$ & $65(43-82)$ & $64(32-83)$ & 0.065 \\
$\quad$ Male & $64 / 93(69)$ & $429 / 577(74)$ & 0.257 \\
$\quad$ Female & $29 / 93(31)$ & $148 / 577(26)$ & \\
Nephrectomy & $74 / 93(79)$ & $474 / 577(82)$ & 0.563 \\
KPS <80\% & $22 / 85(26)$ & $84 / 494(17)$ & 0.067 \\
Diagnosis to treatment & $44 / 93(47)$ & $321 / 573(56)$ & 0.144 \\
$<1$ year & $8 / 68(12)$ & $68 / 398(17)$ & 0.374 \\
Calcium - high & $40 / 82(49)$ & $238 / 487(49)$ & 1.000 \\
Hemoglobin - low & $10 / 80(12)$ & $42 / 504(8)$ & 0.211 \\
Neutrophils - high & $10 / 83(12)$ & $40 / 515(8)$ & 0.200 \\
Platelets - high & & & 0.807 \\
IMDC risk group & $15 / 60(25)$ & $68 / 313(22)$ & \\
$\quad$ Favourable & $33 / 60(55)$ & $183 / 313(58)$ & \\
Intermediate & $12 / 60(22)$ & $62 / 313(20)$ & \\
Poor &
\end{tabular}

IMDC: International Metastatic Renal Cell Carcinoma Database Consortium; IQR: interquartile range; KPS: Karnofsky performance score. predictor of OS for the total cohort (adjusted hazard ratio [aHR] 0.60; 95\% Cl 0.38-0.94). Other significant variables for OS were: low KPS (aHR 2.16; 95\% Cl 1.48-3.15), low hemoglobin (aHR 1.62; 95\% Cl 1.14-2.31), elevated calcium (aHR 1.72; 95\% Cl 1.16-2.56), and time from diagnosis to treatment $<1$ year (aHR $1.72 ; 95 \% \mathrm{Cl} 1.21-2.45$ ).

When analyzed by IMDC risk groups, there was a significant improvement in OS for patients with intermediate-risk disease treated with sunitinib vs. pazopanib (medians 40.1 vs. 20.6 months, $\mathrm{p}=0.014$; aHR 0.50; 95\% Cl 0.27-0.92). There was a numerical improvement in OS with sunitinib vs. pazopanib in the favourable (medians 46.8 vs. 33.8 months; $\mathrm{p}=0.090$ ) and poor-risk groups (medians 12.7 vs. 9.9 months; $\mathrm{p}=0.809$ ); however, these were not statistically significant. Subgroup analysis for higher performance status (KPS $>80 \%$ ) did not reveal significant differences between both groups and analysis by geographic area showed no significant variation by Canadian region, allowing for limited sample sizes.

TTF was also improved for patients treated with sunitinib vs. pazopanib (medians 11.0 vs. 8.4 months, $p=0.130$; aHR $0.87 ; 95 \% \mathrm{Cl} 0.59-1.28)$, although the difference was not statistically significant (Fig. 1B) and this was consistent across IMDC risk groups.

\section{Adverse events}

Overall, common toxicities requiring dose-modification, including fatigue, diarrhea, and hypertension, were simimultivariable analysis, sunitinib use was an independent

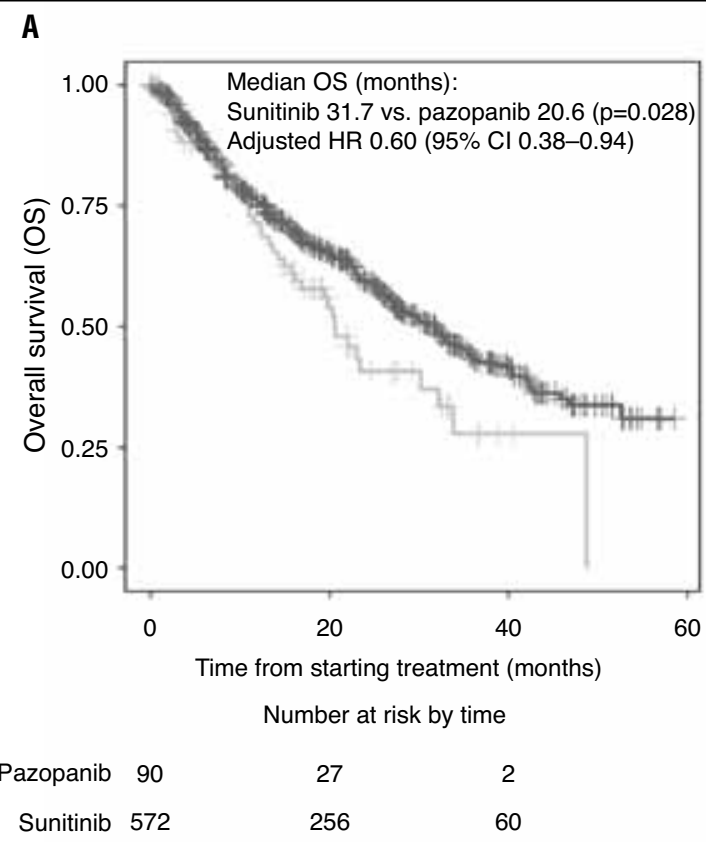

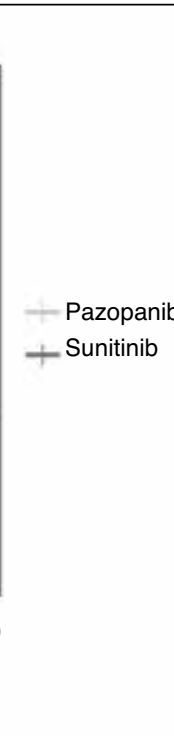

B

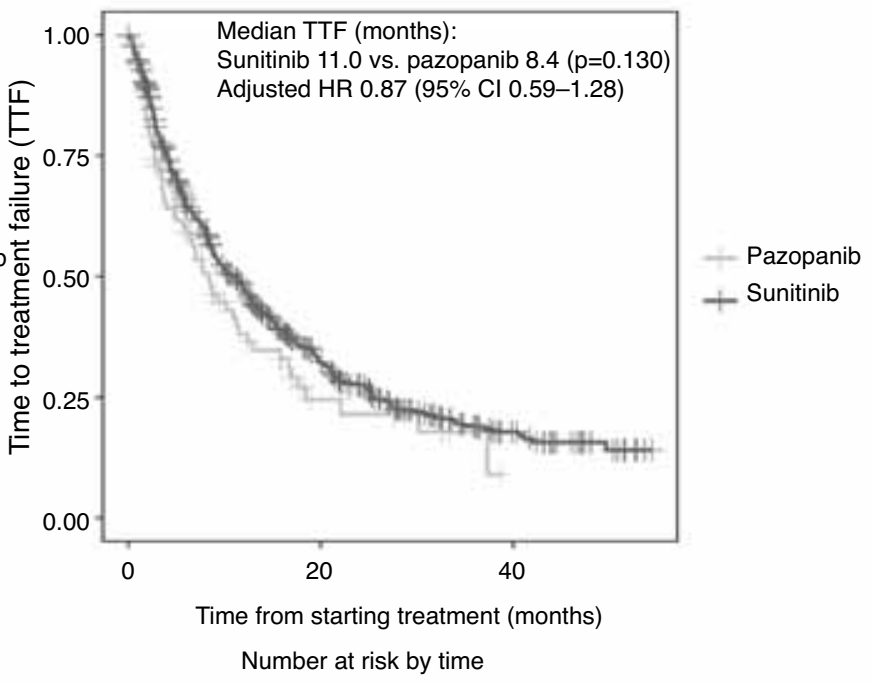

Pazopanib 90

Sunitinib 573

$\begin{array}{cc}9 & 0 \\ 128 & 26\end{array}$

Fig.1. (A) Overall survival (OS) and (B) time-to-treatment failure (TTF) of first-line sunitinib vs. pazopanib. Five sunitinib patients and three pazopanib patients were not included because of missing OS information. Four sunitinib patients and three pazopanib were not included because of missing TTF information; HR: hazard ratio. 


\begin{tabular}{lccc}
\hline Table 2. Dose-modifying adverse events & & \\
\hline Adverse event & $\begin{array}{c}\text { Pazopanib } \\
\text { (n=93) }\end{array}$ & $\begin{array}{c}\text { Sunitinib } \\
(\mathbf{n}=577)\end{array}$ & $\mathbf{p}$ \\
\hline & $\mathrm{n}(\%)$ & $\mathrm{n}(\%)$ & \\
Fatigue & $23(25)$ & $168(29)$ & 0.460 \\
Diarrhea & $16(17)$ & $89(15)$ & 0.650 \\
Mucositis & $7(7)$ & $94(16)$ & 0.028 \\
Hand-foot syndrome & $3(3)$ & $69(12)$ & 0.010 \\
Hypertension & $7(7)$ & $55(9)$ & 0.700 \\
Nausea and vomiting & $9(10)$ & $79(14)$ & 0.320 \\
Gastroesophageal reflux disease & $1(1)$ & $38(7)$ & 0.031 \\
Weight loss & $7(7)$ & $20(4)$ & 0.080 \\
Liver toxicity/altered liver & $13(14)$ & $15(3)$ & 0.001 \\
enzymes* & $2(2)$ & $41(7)$ & 0.100 \\
Thrombocytopenia & $0(0)$ & $21(4)$ & 0.090 \\
Neutropenia & $1(1)$ & $4(<1)$ & 0.530 \\
Anemia &
\end{tabular}

${ }^{*}$ Altered liver enzymes as: increased alanine aminotransferase (ALT), aspartate aminotransferase (AST), and bilirubin.

lar between both groups (Table 2). Patients treated with sunitinib had a significantly higher incidence of mucositis $(16 \%$ vs. $7 \%)$, hand-foot syndrome (HFS, $12 \%$ vs. $3 \%$ ), and gastroesophageal reflux disease (GERD, 7\% vs. 1\%). These patients also had a trend toward more thrombocytopenia and neutropenia. Patients treated with pazopanib had a significantly higher incidence of liver toxicity ( $14 \%$ vs. $3 \%)$ and a trend towards weight loss. Of the patients who discontinued first-line treatment, the proportion that discontinued due to adverse events was $44 \%$ in the pazopanib group and $33 \%$ in the sunitinib group, which was not significantly different $(p=0.306)$.

\section{Discussion}

This is the first population-based analysis evaluating survival outcomes and dose-modifying toxicities in mRCC patients treated with first-line sunitinib or pazopanib. The database used in this analysis contains prospectively collected information and is a valuable resource for evaluating clinical parameters and outcomes seen in the real-world setting. This database includes approximately $1400 \mathrm{mRCC}$ patients treated with surgical and systemic therapies in the modern era.

In our analysis of 670 patients, we demonstrate that, in Canada, patients with mRCC treated with sunitinib appear to have improved OS compared to those treated with pazopanib in the first-line setting. In the pivotal COMPARZ trial, which was statistically designed to be a non-inferiority study, pazopanib was shown to be non-inferior to sunitinib in survival and potentially better-tolerated. ${ }^{4,5}$ However, data comparing these two treatments in a contemporary, population-based setting had been lacking. The centres included in this analysis represent the institutions where the vast majority of patients with $\mathrm{mRCC}$ in Canada are treated and, therefore, the analysis captures the prevailing national practice patterns.

A larger, retrospective initiative by Ruiz-Morales et al. with the IMDC recently showed similar OS (22.3 vs. 22.6 months; $p=0.65$ ) and PFS (8.4 vs. 8.3 months; $p=0.17$ ) between first-line sunitinib and pazopanib. ${ }^{12}$ In our analysis, we show that OS was improved with sunitinib in all risk groups. While this finding was only significant for intermediate-risk patients, there was a trend toward a clinically meaningful improvement for favourable-risk patients as well. Given their prevalence as our largest risk group (Table 1), it likely that the intermediate-risk population is driving our overall OS findings. For the entire cohort, we note that TTF was improved by 2.6 months with sunitinib use. Although this was not statistically significant $(p=0.13)$, this translates to approximately two more cycles of sunitinib therapy, suggesting a potential clinical benefit to patients. Interestingly, the percentages of patients in each of the IMDC risk groups in our study are similar to that seen in the analysis by RuizMorales. This suggests that the CKCis patient population is representative of the wider global perspective, but appears to benefit from a better survival with sunitinib treatment. Perhaps this is related to the fact that the IMDC data did not capture the practice of individualized sunitinib dosing, as Canadian patients make up a minority of the IMDC database and, therefore, the Canadian results may have been diluted out in their overall analysis. We interpret these findings in the context that, in our cohort, potential trends for differences in performance status (KPS) and age are seen in those treated with pazopanib (Table 1). Therefore, it is plausible that these factors of patient selection may impact the observed outcomes, particularly with the improved OS and numerically, but not statistically, improved TTF.

Preclinical and early clinical studies have investigated the potential benefits of individualized dosing of suntinib

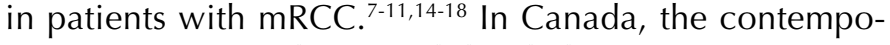
rary experience with sunitinib has led to a practice pattern that incorporates dose and schedule alterations, whereby patients may start on the standard four-weeks-on two-weeksoff schedule, but could also start, or later transition to, an alternate schedule (e.g., two-weeks-on/one-week-off, but also more individualized, such as 10-days-on/seven-daysoff, based on toxicity). This approach of individualized dosing can maintain treatment exposure to sunitinib and potentially limit further toxicities. In our analysis, 19.4\% of the sunitinib cohort started treatment on an alternate, or individualized, schedule. Another 47 patients included in the CKCis database were enrolled in an ongoing phase 2 clinical trial evaluating the efficacy and safety of sunitinib given on an individualized schedule (NCT01499121). ${ }^{19} \mathrm{We}$ also show that subsequent treatment was similar between both groups for approved second-line agents in Canada, which suggests there is no bias in either group in terms of 
exposure to subsequent lines of available therapies. Our analysis, therefore, captures a more contemporary experience with sunitinib use. However, the current investigation does not contain specific outcomes of patients treated with individualized dosing schedules on sunitinb. Further population-based studies analyzing outcomes with this practice pattern are warranted. These real-world studies may add granularity to the impact of dose-individualization on OS and TTF, particularly in the context of other potential confounders previously discussed.

A unique aspect of our study is the data on dose-modifying toxicities. Our analysis confirms known safety signals seen with sunitinib and pazopanib that were similarly demonstrated in the clinical trials that established these therapies. ${ }^{1-6}$ Mucositis, HFS, and GERD were more prominent in the sunitinib group, whereas liver toxicity was seen more in the pazopanib group. Other common toxicities of these therapies, including diarrhea and hypertension, were no different between our cohorts. Notably, patients treated with sunitinib in the COMPARZ trial had higher incidence of Grade 3 or 4 fatigue, ${ }^{4,5}$ whereas in our analysis, the incidence of fatigue was similar between both groups. One possible reason may be the use of contemporary dose and schedule alterations with sunitinib. These individualized adjustments allow for better tolerance of therapy and ongoing exposure to the drug, which may lead to improved efficacy of sunitinib. Interestingly, the proportion of patients who discontinued treatment due to adverse events was numerically higher with pazopanib vs. sunitinib, although this difference was not statistically significant. These proportions in our analysis were greater than those reported in the COMPARZ trial for each drug, ${ }^{4,5}$ which further highlights the differences between adverse events in clinical trials compared to the unselected patient setting. Ultimately, these data on real-world toxicities are informative and may aid physicians and patients in guiding treatment decisions.

Although this is the first population-based database using prospective data to report both survival outcomes and dosemodifying adverse events in $\mathrm{mRCC}$ patients treated with first-line oral VEGF-TT, there are limitations. The time period analyzed, while better reflecting our understanding of toxicity management with VEGF-TT, does have more prevalent use of sunitinib in Canada compared with pazopanib, illustrated by our smaller sample size of patients treated with pazopanib (13.8\%). However, the IMDC analysis by Ruiz-Morales et al had only $12.3 \%$ of their patients treated with pazopanib, ${ }^{12}$ which suggests that our data is representative of pazopanib usage in the real-world setting on a broader, international scale during this time frame. While our patient and disease characteristics were well-balanced and reflective of the typical mRCC population, there was a trend towards poorer performance status (KPS $<80 \%$ ) in the pazopanib group compared with sunitinib ( $26 \%$ vs. $17 \%$; $p=0.067)$. Perhaps this reflects the clinical reality that we tend to offer pazopanib preferentially to patients who are considered fragile or have multiple comorbidities, hence the potential for selection bias. There may also be a trend towards a younger patient population based on the lower end of the age range in the sunitinib group (range 32-83 years); however, there was no statistically significant difference in median age for patients receiving sunitinib vs. pazopanib (median age 64 vs. 65 years; $p=0.065$ ). Overall, our data may simply be a reflection of the practice pattern, patient population, and use of these two therapies in Canada. Specific outcomes in our cohort of dose-individualization on sunitinib are not available currently. However, our findings are hypothesis-generating and the potential of this future data, particularly in the unselected setting, may be revealing. Given these limitations, a specific recommendation about which approved oral VEGF-TT is preferred in the first-line mRCC setting cannot be made based on the results of this analysis.

\section{Conclusion}

We demonstrate that in Canadian patients with mRCC, treatment with sunitinib appears to be associated with an improved OS compared with pazopanib in the first-line setting. Patient selection factors and the contemporary practice of individualized dosing with sunitinib may contribute to these real-world outcomes and warrant further investigations, including studies of individualized dosing with other VEGF-TTs, such as pazopanib. In this regard, ongoing clinical trials will be informative. Furthermore, population-based databases that include survival outcomes and treatmentmodifying toxicities can play a role in guiding therapeutic decisions for physicians and patients in the unselected, community setting.

Competing interests: Dr. Heng has been an advisor for Bayer, BMS, GSK, Janssen, and Pfizer. Dr. Wood has been an advisor for Asetllas, BMS, Novartis, and Pfizer. Dr. Bjarnason has received honoraria from and participated in clinical trials supported by Pfizer. Dr. Kollmannsberger has been an advisor and speaker for Novartis and Pfizer. Dr. Kapoor has been an advisor for Amgen, Astellas, GSK, Janssen, Pfizer, and Sanofi. Dr. Hotte has been an advisor for Astellas and Janssen. Dr. Reaume has been an advisor for GSK, Janssen, Novartis, Pfizer, and Sanofi; and has participated in clinical trials supported by Astellas, BMS, BI, Exelis, GSK, Imclone, Pfizer, and Synta. Dr. Venner has been an advisor for Pfizer; has received payment from Jansen and Novartis, and has participated in clinical trials supported by Astellas, BMS, Lilly, Medivation, and Pfizer. Dr. North has been an advisor for Astellas; has received honoraria from Astellas, Janssen, and Sanofi; and has participated in clinical trials supported by Janssen and Sanofi. Dr. Basappa has been an advisor for Amgen, Astellas, Janssen, Novartis, Pfizer and Trelstar; and has received honoraria from Amgen, Astellas, Janssen, Novartis, and Pfizer. The remaining authors report no competing personal or financial interests.

Acknowledgment: This project was partially supported by the Kidney Cancer Research Network of Canada (KCRNC) 
This paper has been peer-reviewed.

\section{References}

1. Motzer RJ, Hutson TE, Tomczak P, et al. Sunitinib vs. interferon alfa in metastatic renal-cell carcinoma. N Engl J Med 2007;356:115-24. https://doi.org/10.1056/NEJMoa065044

2. Motzer RJ, Hutson TE, Tomczak $P$, et al. Overall survival and updated results for sunitinib compared with interferon alfa in patients with metastatic renal cell carcinoma. J Clin Oncol 2009;27:3584-90. https://doi.org/10.1200/JC0.2008.20.1293

3. Sternberg CN, Davis ID, Mardiak J, et al. Pazopanib in locally advanced or metastatic renal cell carcinoma: Results of a randomized, phase 3 trial. J Clin Oncol 2010;28:1061-8. https://doi.org/10.1200/ JC0.2009.23.9764

4. Motzer RJ, Hutson TE, Cella D, et al. Pazopanib vs. sunitinib in metastatic renal cell carcinoma. N Engl J Med 2013;369:722-31. https://doi.org/10.1056/NEJMoal303989

5. Motzer RJ, Hutson TE, McCann L, et al. Overall survival in renal cell carcinoma with pazopanib vs. sunitinib. N Engl J Med 2014;370:1769-70. https://doi.org/10.1056/NEJMc1400731

6. Escudier B, Porta C, Bono P, et al. Randomized, controlled, double-blind, crossover trial assessing treatment preference for pazopanib vs. sunitinib in patients with metastatic renal cell carcinoma: PISCES study. J Clin Oncol 2014;32:1412-8. hitps://doi.org/10.1200/JC0.2013.50.8267

7. Faivre S, Delbaldo C, Vera K, et al. Safety, pharmacokinetic, and antitumour activity of SU1 1248, a novel oral multitarget tyrosine kinase inhibitor, in patients with cancer. J Clin Oncol 2006;24:25-35. https://doi.org/10.1200/JC0.2005.02.2194

8. Chow LQM, Eckhardt SG. Sunitinib: From rational design to clinical efficacy. J Clin Oncol 2007;25:884-96 https://doi.org/10.1200/JC0.2006.06.3602

9. Escudier B, Roigas J, Gillessen S, et al. Phase 2 study of sunitinib administered in a continuous once-daily dosing regimen in patients with cytokine-refractory metastatic renal cell carcinoma. J Clin Oncol 2009; 27:4068-75. https://doi.org/10.1200/JC0.2008.20.5476

10. Barrios $\mathrm{CH}$, Hernandez-Barajas $\mathrm{D}$, Brown $M$ P, et al. Phase 2 trial of continuous once-daily dosing of sunitinib as first-line treatment in patients with metastatic renal cell carcinoma. Cancer 2012;118:1252-9. https://doi.org/10.1002/cncr.26440
11. Najiar YG, Mittal K, Elson P, et al. A two-weeks-on and one-week-off schedule of sunitinib is associated with decreased toxicity in metastatic renal cell carcinoma. Eur J Cancer 2014;50:1084-9. https://doi.org/10.1016/i.eica.2014.01.025

12. Morales JMR, Swierkowski M, Wells JC, et al. First-line sunitinib vs. pazopanib in metastatic renal cell carcinoma: Results from the international metastatic renal cell carcinoma database consortium. Eur J Cancer 2016;65:102-8. https://doi.org/10.1016/i.eica.2016.06.016

13. Heng D, Xie W, Regan M, et al. External validation and comparison with other models of the International Metastatic Renal-Cell Carcinoma Database Consortium prognostic model: A population-based study. Lancet Oncol 2013;14:141-8. https://doi.org/10.1016/S1470-2045(12)70559-4

14. Atkinson BJ, Kalra $S$, Wang $X$, et al. Clinical outcomes in patients with metastatic renal cell carcinoma treated with alternative sunitinib schedules. J Urol 2014;191:611-8. https://doi.org/10.1016/i. juro.2013.08.090

15. Bjarnason GA, Khalil B, Hudson JM, et al. Outcomes in patients with metastatic renal cell cancer treated with individualized sunitinib therapy: Correlation with dynamic microbubble ultrasound data and review of the literature. Urol Oncol 2014;32:480-7. hrtps://doi.org/10.1016/i.urolonc.2013.10.004

16. Neri $B$, Vannini $A$, Brugia $M$, et al. Biweekly sunitinib regimen reduces toxicity and retains efficacy in metastatic renal cell carcinoma: A single-centre experience with 31 patients. Int I Urol 2013;20:478-83. https://doi.org/10.1111/i.1442-2042.2012.03204.x

17. Kondo T, Takagi T, Kobayashi $\mathrm{H}$, et al. Superior tolerability of altered dosing schedule of sunitinib with two-weeks-on and one-week-off in patients with metastatic renal cell carcinoma-comparison to standard dosing schedule of four-weeks-on and two-weeks-off. Jpn I Clin Oncol 2014;44:270-7. https://doi.org/10.1093/iico/hyt232

18. Kalra S, Rini BI, Jonasch E. Alternate sunitinib schedules in patients with metastatic renal cell carcinoma. Ann Oncol 2015;26:1300-4. https://doi.org/10.1093/annonc/mdv030

19. Bjarnason $\mathrm{GA}$, Knox JJ, Kollmannsberger $\mathrm{CK}$, et al. Phase 2 study of individualized sunitinib as first-line therapy for metastatic renal cell cancer (mRCC). J Clin Oncol 2015;33:S4555.

Correspondence: Dr. Naveen S. Basappa, Cross Cancer Institute, Edmonton, AB, Canada; Naveen.Basappa@Albertahealthservices.ca 